\title{
Efecto de la Temperatura y Tiempo de Almacenamiento sobre la Calidad del Tomate Deshidratado
}

Betty A. Ronceros, Javier I. Leiva, Elizabeth del C. Burgos y Lorena del C. Pardo Universidad de Los Lagos, Dpto. de Ciencia y Tecnología de los Alimentos, Avenida Fuchslocher 1305, Casilla 933, Osorno-Chile (e-mail: bronceros@ulagos.cl)

\section{Resumen}

El objetivo de este estudio fue evaluar el efecto de la temperatura y tiempo de almacenamiento sobre la calidad microbiológica y sensorial de tomates deshidratados. Se determinó el contenido de humedad y actividad de agua, se realizó recuento de Escherichia coli, hongos y levaduras, y se evaluó la intensidad percibida en cada característica sensorial del tomate deshidratado. La información fue analizada mediante determinación de efectos, coeficientes de correlación, regresión múltiple, análisis de componentes principales y análisis de conglomerados. Los resultados indicaron una asociación importante entre hongos, levaduras y Escherichia coli, además de apariencia, aroma y sabor. Basado en los resultados del estudio, se concluye que el tomate deshidratado presentó características microbiológicas y sensoriales deseables a temperaturas entre 7 y $20^{\circ} \mathrm{C}$ por un tiempo de hasta 60 días.

\section{Effect of Temperature and Storage Time on the Quality of the Dehydrated Tomatoes}

\begin{abstract}
The objective of this study was to evaluate the effect of temperature and storage time on the microbiological and sensorial quality of dehydrated tomatoes. Moisture content and water activity were determined, Escherichia coli, fungal and yeast counts were performed, and the intensity of each sensorial property of the dehydrated tomato was evaluated. Information obtained was analyzed by determination of effects, correlation coefficients, multiple regression, main components and cluster analysis. The results showed an important association between fungal, yeast and Escherichia coli, besides appearance, odor and flavor. Based on the results of this study, it concluded that the dehydrated tomato presented desirable microbiological and sensorial characteristics at temperatures between 7 and $20^{\circ} \mathrm{C}$ for a period up to 60 days.
\end{abstract}

Keywords: dehydrated tomato, microbiological quality, sensorial quality, storage time 


\section{INTRODUCCIÓN}

La deshidratación del tomate es un proceso usado para preservar y extender su vida útil. La calidad del alimento deshidratado es a menudo inferior. El colapso de la estructura, pérdida de textura, desarrollo microbiano y decoloración son problemas frecuentes de calidad. Por lo mismo, se hace necesario controlar estos factores, con el propósito de minimizar su impacto sobre la calidad y garantizar la vida útil del alimento (Watada et al., 1990; Wiley, 1997; Heredia et al., 2007). Aunque la deshidratación de frutos y vegetales ha sido investigada intensivamente (Yang et al., 1987; Litvin et al., 1998; Stojanovic y Silva, 2007; Taiwo et al., 2007), pocos estudios han investigado el efecto de las condiciones de almacenamiento sobre la calidad del alimento (Prothon et al., 2003).

Khin et al. (2007), estudiando la transferencia de masa en manzana deshidratada, indicaron que los parámetros texturales: dureza, fragilidad, elasticidad y cohesividad, sufren modificaciones después del secado con aire a $70^{\circ} \mathrm{C}$ por $10 \mathrm{~min}$. Allan-Wotjas et al. (1999), estudiando el efecto de las bajas temperaturas de almacenamiento sobre la microestructura del arándano, encontraron que la calidad fisicoquímica más adecuada, en cuanto a bayas dañadas, resultó de índices de congelación rápidos y de temperaturas bajas de almacenamiento. Inatsu et al. (2007), evaluaron la eficacia del saneamiento con clorito de sodio durante el prelavado de coles. Los mismos autores, indicaron que el tratamiento disminuyó el nivel de patógenos y que no se observó ninguna diferencia significativa en color, olor y textura al octavo día de almacenamiento a $10^{\circ} \mathrm{C}$. Sin embargo, dichos investigadores encontraron que el contenido de L. monocytogenes después del prelavado con clorito de sodio aumentó gradualmente con el tiempo de almacenamiento.

Los resultados encontrados por Varoquaux et al. (1990), mostraron que las rodajas de kiwi pierden un $50 \%$ de su firmeza inicial antes de 2 días de almacenamiento a $2^{\circ} \mathrm{C}$, causado por la hidrólisis enzimática de los componentes de la pared celular. Iturriaga et al. (2007), encontraron que almacenar tomate durante 10 días a $30^{\circ} \mathrm{C}$ fue la causa de que la población de Salmonella aumentará en $86 \%$. Los mismos autores indican la importancia de mantener los tomates almacenados a temperaturas y humedades que no promuevan el crecimiento de bacterias patógenas.

La metodología empleada en la presente investigación consistió en medir características microbiológicas y sensoriales a diferentes tiempos y temperaturas de almacenamiento. El objetivo de este estudio fue determinar el efecto de la temperatura y tiempo de almacenamiento sobre la calidad microbiológica y sensorial del tomate deshidratado.

\section{MATERIALES Y MÉTODOS}

Se utilizaron tomates (Lycopersicon esculentum var. pyriforme) cultivados en la Provincia de Talca (VII Región, Chile) cuyo contenido de humedad inicial fue de 94,5\%.

Los tomates fueron lavados por aspersión, seleccionados por tamaño, eliminado el cádiz y pedúnculo, trozados en rodajas $(7 \pm 1 \mathrm{~mm}$ de espesor) y deshidratados en secador de bandeja a $58^{\circ} \mathrm{C}$ por $8,5 \mathrm{~h}$.

El tomate deshidratado fue almacenado a diferentes temperaturas $\left(7,20\right.$ y $\left.30^{\circ} \mathrm{C}\right)$ y tiempos $(30,60$ y 90 días) tratando de simular las condiciones que enfrenta el producto en el hogar o en un lugar de venta. Cumplidas las condiciones de almacenamiento, se controló la humedad (hum) y actividad de agua $\left(\mathrm{a}_{\mathrm{w}}\right)$ según el método propuesto por Guarda y Álvarez (1991). La calidad microbiológica se controló mediante recuento de E. coli (Ecoli), hongos (hon) y levaduras (lev) según la metodología indicada por APHA (1992). La evaluación sensorial la realizaron 12 panelistas semientrenados. Se usó una prueba de escala hedónica (no estructurada) según la metodología descrita por Anzaldúa-Morales (1994). En el análisis se emplearon escalas crecientes de 0 a 15 para expresar la intensidad (0=disgusta, 15=gusta) percibida para cada atributo organoléptico: apariencia (apa), color (col), aroma (aro), sabor (sab) y textura (text). 
Los resultados fueron evaluados por determinación de efectos (mediante rango percentil), coeficientes de correlación, regresión múltiple, análisis de componentes principales y análisis de conglomerados, usando el programa computacional Statistica 6,0.

\section{RESULTADOS Y DISCUSIÓN}

El efecto para cada una de las características de calidad del tomate deshidratado se presenta en la Figuras 1,2 y 3.

En la Figura 1 se observa que a los 30 días hubo una mayor presencia de lev y hon. La explicación de aquello, puede deberse a la mayor asociación existente entre éstos microorganismos y la $a_{w}$, mostrando una menor correlación con la hum (Tabla 1). En general, las características sensoriales mostraron un bajo efecto sobre la calidad del producto. Sin embargo, a $20^{\circ} \mathrm{C}$, la text mostró una presencia importante. La razón puede ser la mayor pérdida de hum que se alcanza al haber una alta presencia de ésta. Sanjuan et al. (2004), indican que la firmeza del brócoli deshidratado aumenta con el incremento de la temperatura de almacenamiento.

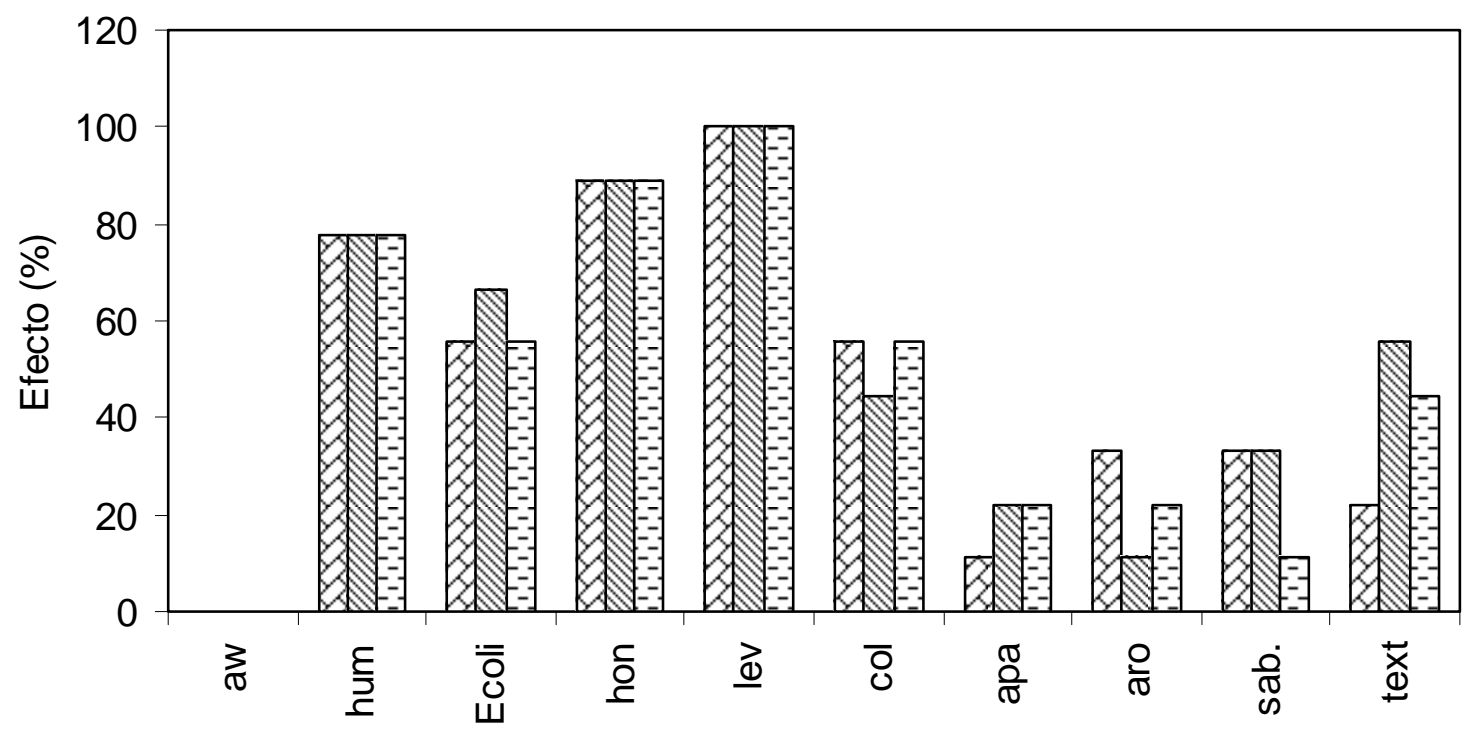

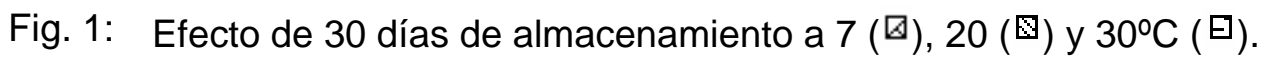

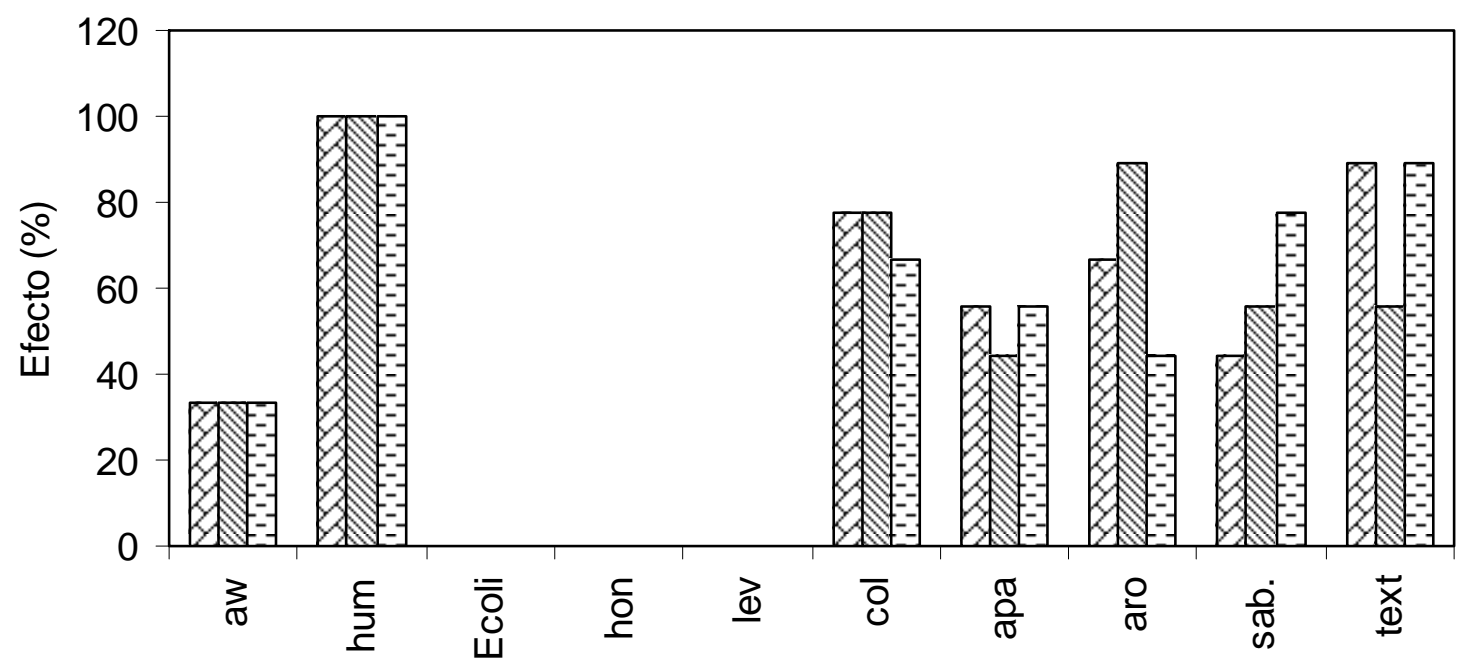

Fig. 2: Efecto de 60 días de almacenamiento a $7\left({ }^{\circledR}\right), 20\left({ }^{\mathbb{Q}}\right)$ y $30^{\circ} \mathrm{C}\left({ }^{\Xi}\right)$. 
En la Figura 2 se observa que las características microbiológicas no presentaron un efecto sobre la calidad del producto, por tal motivo, no se representaron barras para dichas características. Marth y Steel (1998), manifiestan que ésta ausencia se debe a que a valores de actividad de agua inferiores a 0,95 y 0,80 (humedad cercana al 15\%, en este último caso), casi todos los hongos y levaduras junto con bacterias como $E$. coli se encuentran inhibidos, respectivamente.

En la Figura 3 se observa que la hum y las características microbiológicas se mostraron altas a los 90 días de almacenamiento, probablemente debido a la condensación de calor en la superficie del alimento. Según lo reportado por Hertog et al. (2004), la alta humedad propició la degradación de estructura y liberación de componentes aromáticos en el producto. Esto significa que el tomate deshidratado perdería firmeza y otros atributos sensoriales relevantes al prolongar el almacenamiento más allá de los 90 días, especialmente a los $20^{\circ} \mathrm{C}$.

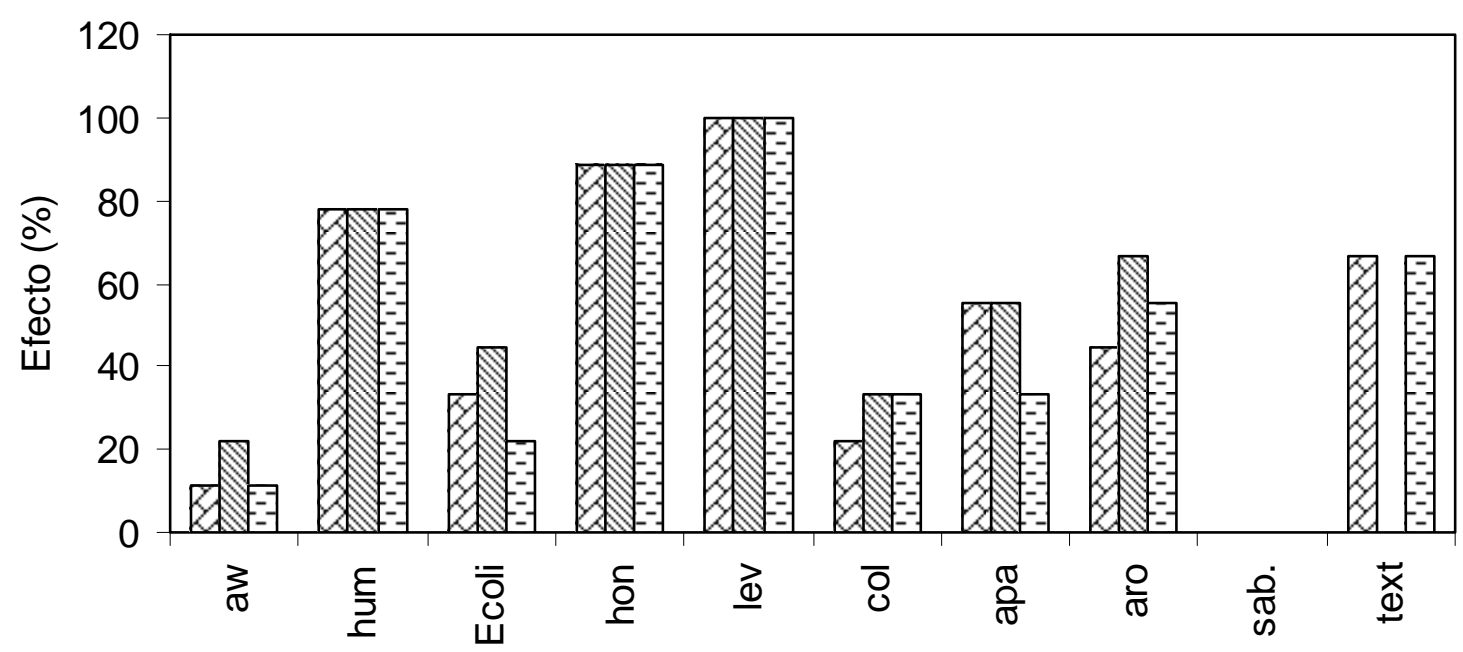

Fig. 3: Efecto de 90 días de almacenamiento a $7\left({ }^{\circledR}\right), 20$ (\$) y $30^{\circ} \mathrm{C}\left({ }^{\boxminus}\right)$.

Los coeficientes de correlación $(r)$ de las características sensoriales y microbiológicas se presentan en la Tabla 1. Las correlaciones marcadas en cursiva indican diferencia significativa a $p<0,05$.

Se observaron correlaciones positivas y negativas altamente significativas a un nivel de $p<0,05$ entre: hon y lev, Ecoli con hon, lev y sab, y entre apa con aro y sab. De acuerdo a estos resultados, reviste importancia la estrecha asociación entre hon, lev y Ecoli, además de apa, aro y sab como caracteres relevantes de la calidad microbiológica y sensorial, respectivamente. Oladiran e Iwu (1993), comentan que la putrefacción del tomate durante su almacenamiento se debe principalmente al desarrollo de los hongos Geotrichum candidum y Aspergillus flavus.

La correlación encontrada entre text y col fue cercana a 0,5. Sanjuan et al. (2004), manifiestan que la firmeza es una variable relevante de la calidad sensorial del brócoli deshidratado. Heredia et al. (2007), comentan que en el tomate deshidratado, los problemas frecuentes de calidad son: colapso estructural, pérdida de textura y flavor, y decoloración.

La $a_{w}$ mostró una correlación mayor con los atributos sensoriales, principalmente, col y text. Lo anterior, coincide con lo reportado por Akanbi et al. (2006), los cuales indican que los cambios sensoriales en rodajas de tomate deshidratado dependen fuertemente del contenido de actividad de agua.

La representación gráfica de las coordenadas según el análisis de componentes principales se presenta en la Figura 4; en esta figura se observó en un plano cartesiano, la ubicación de las 10 características utilizando los 2 primeros factores como ejes de abscisas y ordenadas. Las características que son mejor explicadas por el eje 1 y 2 están concentradas hacia la parte horizontal y vertical del plano, respectivamente. 
Tabla 1: Coeficientes de correlación entre las características sensoriales y microbiológicas.

\begin{tabular}{|l|c|c|c|c|c|c|c|c|c|c|}
\hline & $\mathrm{a}_{\mathrm{w}}$ & hum & Ecoli & hon & lev & col & apa & aro & sab & text \\
\hline $\mathrm{a}_{\mathrm{w}}$ & 1,00 & & & & & & & & & \\
hum & 0,66 & 1,00 & & & & & & & & \\
Ecoli & 0,20 & 0,01 & 1,00 & & & & & & & \\
hon & 0,37 & 0,29 & 0,83 & 1,00 & & & & & & \\
lev & 0,33 & 0,16 & 0,97 & 0,86 & 1,00 & & & & & \\
col & $-0,46$ & $-0,54$ & $-0,42$ & $-0,39$ & $-0,54$ & 1,00 & & & & \\
apa & 0,13 & $-0,42$ & 0,13 & 0,18 & 0,03 & 0,31 & 1,00 & & & \\
aro & 0,03 & $-0,28$ & 0,05 & 0,05 & $-0,08$ & 0,57 & 0,77 & 1,00 & & \\
sab & $-0,38$ & 0,17 & $-0,69$ & $-0,54$ & $-0,61$ & 0,14 & $-0,71$ & $-0,54$ & 1,00 & \\
text & $-0,40$ & $-0,39$ & $-0,30$ & $-0,16$ & $-0,41$ & 0,47 & 0,14 & 0,04 & 0,09 & 1,00 \\
\hline
\end{tabular}

Las características sensoriales: apa, aro, text y col se agruparon muy próximas entre sí debido principalmente a la estrecha asociación entre ellas (Tabla 1). Destaca la ubicación casi opuesta del grupo: Ecoli, hon, lev, $a_{w}$ y hum. El sab mostró una relación significativa tanto con apa como con Ecoli. Este vínculo, permitiría manejar en conjunto la calidad microbiológica y sensorial del tomate deshidratado.

En cuanto a la ubicación cercana de las características al círculo de correlación o circunferencia, los resultados del trabajo se corresponden a lo esperado según Phillippeau (1990) y Cuadras (2002).

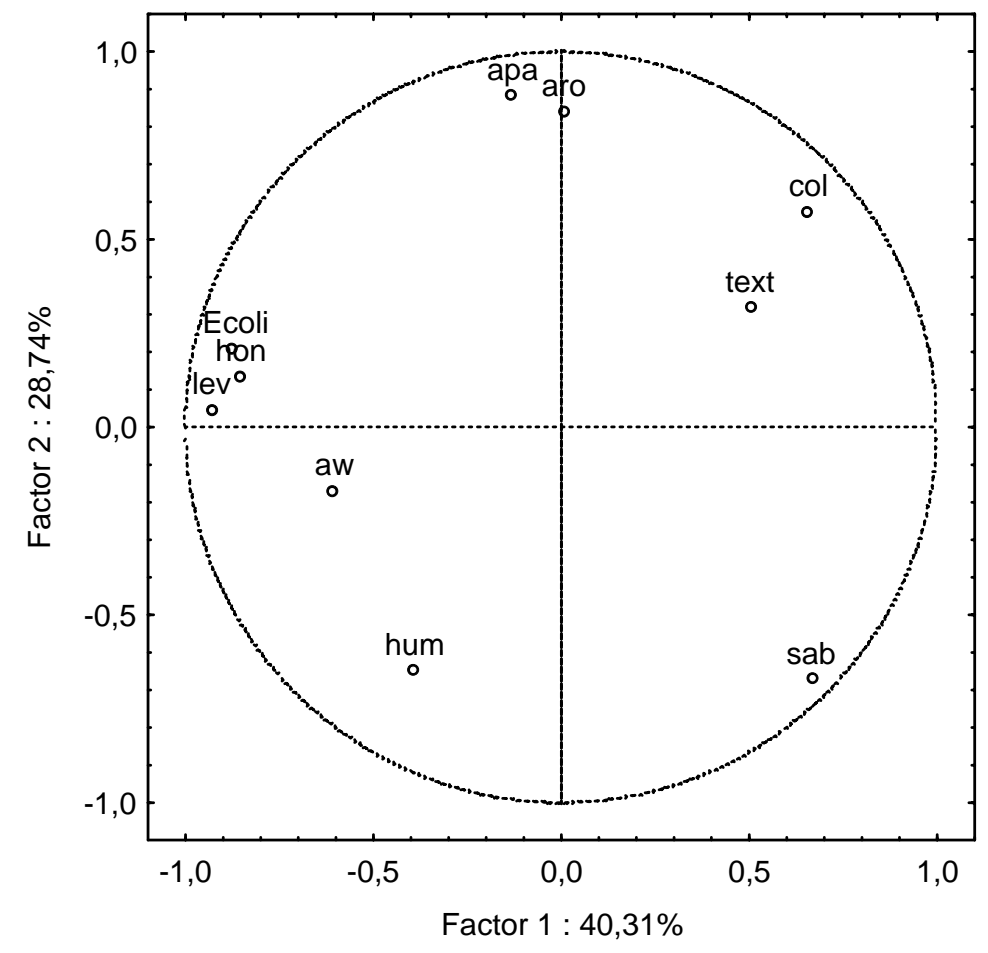

Fig. 4: Representación de las características sensoriales y microbiológicas del tomate deshidratado en los ejes factoriales 1 y 2.

En el dendograma obtenido (Figura 5) y considerando como punto de corte el nivel correspondiente a 4, se observa que con las 10 características se forman 2 grandes grupos. El primero conformado por los subgrupos: apa, aro, text y col además de sab, destacando la proximidad entre las dos primeras como consecuencia de la asociación altamente significativa entre ambas. El segundo grupo estuvo conformado por los subgrupos: hum, $a_{w}$, Ecoli, y el conjunto de lev y hon, las tres últimas características microbiológicas muy próximas entre sí debido principalmente a la estrecha asociación entre ellas (Figura 4). 
De ello, se deduce que considerando cuatro características, una de cada subgrupo, se puede predecir la calidad microbiológica y sensorial del tomate deshidratado, equivalente a lo que se obtendría considerando la totalidad de las variables analizadas inicialmente.

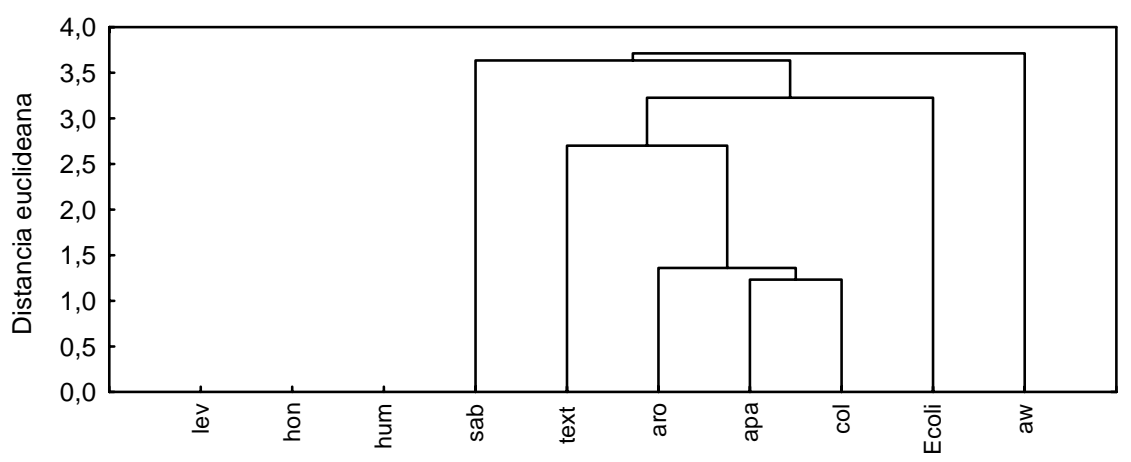

Fig. 5: Dendograma de las características sensoriales y microbiológicas.

A través de la regresión múltiple, se pudo predecir el sab a partir de algunas características sensoriales y microbiológicas, entre ellas, apa además de Ecoli, hon y lev, respectivamente. Los coeficientes de regresión múltiple son mostrados en la Tabla 2.

Tabla 2: Coeficientes de regresión múltiple para sab en función de las características

\begin{tabular}{|l|r|r|c|}
\multicolumn{2}{c}{ Sensoriales y microbiológicas (*, significativo. NS, no significativo). } \\
\hline Características & Coeficiente & $\begin{array}{c}\text { Desviación } \\
\text { estándar }\end{array}$ & $\begin{array}{c}\text { Significancia } \\
(p<0,03)\end{array}$ \\
\hline Ecoli & $-0,58$ & 0,82 & $\mathrm{NS}$ \\
hon & $2,76 \cdot 10^{-3}$ & $3,20 \cdot 10^{-3}$ & $\mathrm{NS}$ \\
lev & $-3,66 \cdot 10^{-6}$ & $12,90 \cdot 10^{-5}$ & $\mathrm{NS}$ \\
apa & $-1,70$ & 0,49 & * \\
Intercepto & 5,05 & 0,88 & * \\
\hline
\end{tabular}

Por lo tanto, la ecuación de regresión es:

$$
\mathrm{sab}=5,05-0,58 \text { Ecoli }+\left(2,76 \cdot 10^{-3}\right) \text { hon }-\left(3,66 \cdot 10^{-6}\right) \text { lev }-1,70 \text { apa } \quad R=0,94
$$

R representa el coeficiente de correlación múltiple entre la variable dependiente y el conjunto de variables independientes. Esto significa que el $94 \%$ de la variación en porcentaje de sab, se explica mediante el modelo de regresión lineal obtenido. Se nota, por la ecuación (1), que cuanto mayor es la apa (característica sensorial), menor es el valor de sab. Del mismo modo, al aumentar el valor de E.coli (característica microbiológica), disminuye el sab, aunque en menor grado éste último.

\section{CONCLUSIONES}

De acuerdo a los resultados obtenidos y analizados en la presente investigación, se concluye lo siguiente:

A través de este estudio, se pudieron clasificar las características de calidad del tomate deshidratado por su variabilidad sensorial y microbiológica. Estas características pudieron ser claramente diferenciadas y agrupadas en 4 subgrupos, entre ellas.

La calidad microbiológica presentó un comportamiento inversamente proporcional a la calidad sensorial, y en ésta última, se observó un comportamiento idéntico entre sabor y apariencia. La presencia de hongos y levaduras no tuvo un efecto significativo sobre la calidad sensorial. El 
atributo sensorial sabor representó un puente de unión entre las calidades estudiadas, o sea, conociendo éste atributo, se podría realizar una estimación razonable de ambas calidades.

El tomate deshidratado presentó un buen comportamiento microbiológico y sensorial a temperaturas entre 7 y $20^{\circ} \mathrm{C}$ por un tiempo de hasta 60 días.

La regresión múltiple permitió obtener una ecuación de predicción del comportamiento del sabor en relación a la presencia de E. coli, hongos, levaduras y apariencia en el tomate deshidratado.

\section{REFERENCIAS}

Akanbi, C.T., R.S. Adeyemi y A. Ojo; Drying characteristics and sorption isotherm of tomato slices, J. Food Eng.: 73(2), 157-163 (2006).

Allan-Wotjas, P., H.D. Goff y R. Stark; The effect of freezing method and frozen storage conditions on the microstructure of wild blueberries as observed by cold-stage scanning electron microscopy, Scanning: 21(5), 334-347 (1999).

Anzaldúa-Morales, A.; La evaluación sensorial de los alimentos en la teoría y la práctica, Editorial Acribia, España (1994).

APHA; American Public Health Association, Compendium of methods for the microbiological examination of foods, $16^{\text {th }}$ edition, Washington D.C. (1992).

Cuadras, C.; Métodos de análisis multivariante, Editorial EUB, S.L., Barcelona, España (2002).

Guarda, A. y P.I. Álvarez; Evaluación estadística de un método rápido y sencillo para determinar la actividad del agua en alimentos, Alimentos: 16(3), 9-13 (1991).

Heredia, A., C. Barrera y A. Andres; Drying of cherry tomato by a combination of different dehydration techniques. Comparison of kinetics and other related properties, J. Food Eng.: 80(1), 111-118 (2007).

Hertog, M.L., R. Ben-Arie, E. Roth y B.M. Nicolai; Humidity and temperature effects on invasive firmness measures, Postharvest Biol. Tec.: 33(1), 79-91 (2004).

Inatsu, Y., M.L. Bari y S. Kawamoto; Application of acidified sodium chlorite prewashing treatment to improve the food higiene of lightly fermented vegetables, Jarq-Jpn Agr. Res. Q.: 41(1), 17-23 (2007).

Iturriaga, M.H., M.L. Tamplin y E.F. Escartin; Colonization of tomatoes by Salmonella Montevideo is affected by relative and storage temperature, J. Food Prot.: 70(1), 30-34 (2007).

Khin, M.M., W.B. Zhou y S.Y. Yeo; Mass transfer in the osmotic dehydration of coated apple cubes by using maltodextrin as the coating material and their textural properties, J. Food Eng.: 81(3), 514-522 (2007).

Litvin, S., C.H. Mannheim y J. Miltz; Dehydration of carrots by a combination of freeze drying, microwave heating and air or vacuum drying, J. Food Eng.: 36(1), 103-111 (1998).

Marth, E.H. y J.L. Steel; Applied Dairy Microbiology. Marcel Dekker Inc., New York (1998).

Oladiran, A.O. y L.N. Iwu; Studies on the fungi associated with tomato fruti rots and effects of enviroment on storage, Mycopathologia: 121(3), 157-161 (1993).

Philippeau, G.; ¿Cómo interpretar los resultados de un análisis de componentes principales?, Service dus Eludes Statistiques, Francia (1990). 
Prothon, F., L. Ahrne y I. Sjoholm; Mechanisms and prevention of plant tissue collapse during dehydration: a critical review, Crit. Rev. Food Sci.: 43(4), 447-479 (2003).

Sanjuan, N., J. Bon, G. Clement y A. Mulet; Changes in the quality of dehydrated broccoli florets during storage, J. Food Eng.: 62(1), 15-21 (2004).

Stojanovic, J. y J.L. Silva; Influence of osmotic concentration, continuous high frequency ultrasound and dehydration on antioxidants, colour and chemical properties of rabbiteye blueberries, Food Chem.: 101(3), 898-906 (2007).

Taiwo, K.A., O.D. Baik y A.O. Farinu; Kinetics of heat and mass transfer and color development of pre-treated sweet potatoes during frying, T. Asae: 50(1), 129-135 (2007).

Varoquaux, P., I. Lecendre, F. Varoquaux y M. Souty; Change in firmness of kiwi fruit after slicing, Sci. Alim.: 10, 127-139 (1990).

Watada, A.E., K. Abe y N. Yamauchi; Physiological activities of partially processed fruits and vegetables, Food Technol.: 40(5), 116-122 (1990).

Wiley; R.; Frutas y hortalizas mínimamente procesadas y refrigeradas, Editorial Acribia, España (1997).

Yang, A.P.P., C. Wills y T.C.S. Yang; Use of a combination process of osmotic dehydration and freeze-drying to produce a raisin-type lowbush blueberry product, J. Food Sci.: 52(6), 1651 (1987). 